\title{
Communicable Diseases Report, NSW, May and June 2008
}

\section{Communicable Diseases Branch, NSW Department of Health}

For updated information, including data and facts on specific diseases, visit www.health.nsw.gov.au and click on Infectious Diseases.

The communicable diseases site is available at: http://www.health.nsw.gov.au/publichealth/ infectious/index.asp.

Tables 1 and 2, and Figure 1 show reports of communicable diseases received through to the end of June 2008 in New South Wales (NSW).

\section{Measles}

From January to June 2008, 38 cases of measles were reported in NSW compared with four cases reported in 2007. The majority of cases were reported from public health units in the Sydney area.

Fourteen cases were notified in May and a further three confirmed in June. Of those 17 cases, two were associated with overseas travel, 10 were associated with a single cluster in south-west Sydney and one had contact with a known case; for the remaining cases, the source of infection was not identified. The cases ranged in age from 7 months to 36 years; 10 were male and seven female. Over half the cases had not been immunised against measles.

In the cluster of 10 cases identified in south-west Sydney in May and early June, the initial case had attended an emergency department but was not diagnosed with measles at the time. Two siblings of this case (thought to be fully vaccinated against measles) also presented to the same emergency department five days later with fever, cough and rash (consistent with measles). The cluster had three generations of transmission:

- Generation 1: the initial case and the initial case's siblings (who were likely infected at the same time from an unknown source)

- Generation 2: four of their emergency department contacts and one of their school contacts

- Generation 3: one of the emergency department contact's work colleagues (resident in west Sydney) and one in a family member of the school contact.

Public health units across Sydney collaborated to investigate this outbreak and conducted clinics for contacts of the cases to offer vaccination and provide information.

\section{Tuberculosis}

There are close to 450 cases of tuberculosis notified every year in NSW. Treatment of the affected person and followup of any contacts occur primarily through the network of chest clinics across the state.

In May and June, two large school-based screenings were undertaken by Sydney South West Area Health Service.

\section{Case 1}

NSW Health was notified of a case of tuberculosis in a young man who had attended a school in the inner west of Sydney. He was symptomatic in October 2007, but not diagnosed with tuberculosis and did not commence treatment until December while overseas. The diagnosis was not reported to NSW Health until he returned to Australia some months later.

Tuberculin skin test results from the case's closest contacts (i.e. those in his household) suggested that he was unlikely to have been very infectious while at school or in flight. However, the area health service contacted the school administration and with its assistance identified 45 students and five teachers who may have been in close contact with the case. Tuberculin skin tests to screen for possible latent tuberculosis infection were offered. Follow-up continues.

\section{Case 2}

In early June, a young man attending a special school in south-west Sydney was diagnosed with active tuberculosis. Almost 50 students and staff are undergoing assessment for possible transmission. Follow-up continues.

\section{Meningococcal disease}

There were 11 cases of meningococcal disease in NSW residents in June. Seven of these were caused by Neisseria meningitidis serogroup $\mathrm{B}$, for which there is no effective vaccine at present. Of the 11 cases:

- four were pre-schoolers, three were school-aged children, and four were adults

- seven were female 
- five lived in metropolitan, three in regional and three in rural locations.

Children in NSW are routinely offered vaccination against meningococcal disease from the age of 12 months. In recent years, a high school catch-up immunisation program has been conducted throughout the state. This conjugated vaccine only protects against serogroup $\mathrm{C}$ disease.

The causative agent is spread in the fine droplets shed through coughing, sneezing and spluttering. The risk from saliva from the front of the mouth is not as important as once thought.

In all cases, close contacts are assessed for the need for clearance antibiotics in case they may be carriers. Advice is given about disease symptoms and the need to seek urgent medical advice if unwell.

Control measures in NSW are based on current national guidelines, available at http://www.health.gov.au/internet/ main/publishing.nsf/Content/cda-pubs-other-mening2007.htm

\section{Influenza}

Enhanced surveillance for influenza continued in May and June in the expectation of increased numbers of cases. This surveillance tracks data from four sources:

- influenza-like illness presentations to 28 emergency departments across NSW

- laboratory diagnoses of respiratory infections

- deaths due to influenza or pneumonia

- outbreaks.

During May and June, influenza data indicated normal background influenza activity for the season. A total of 34 laboratory-confirmed cases were notified in the state in May and 45 in June.

While vaccination against influenza may cause some side effects (mild fever, muscle aches) the vaccine does not contain live virus, and cannot cause true influenza.

\section{Enteric diseases}

In May and June 2008, NSW public health units investigated 95 outbreaks of gastroenteritis, including 88 suspected to be caused by person-to-person spread, and seven suspected to be foodborne. Foodborne outbreaks are investigated in collaboration with the NSW Food Authority.

The 88 suspected person-to-person outbreaks affected a total of 1245 people. Fifty-two occurred in aged-care facilities and affected 779 people; 22 occurred in hospitals and affected 283 people; 12 occurred in child-care centres and affected 103 people; one was in a community centre affecting three people; and one in a school where 77 students were reported absent.

Clinical specimens were submitted for testing for 39 of the 88 suspected person-to-person gastroenteritis outbreaks. Rotavirus was confirmed in stool samples from one agedcare facility outbreak, and norovirus was identified in 13 outbreaks. The causative agent was not confirmed for the remaining outbreaks.

Of the seven suspected foodborne gastroenteritis outbreaks, one was of likely viral origin affecting 17 people, where an epidemiological investigation indicated the vehicle was roast pork served at a catered function. One outbreak in a correctional facility affected all 14 people who shared a common meal; the pathogen was not identified. Chinese banquet style meals from two different restaurants were implicated in two small outbreaks with 12 people affected. Two other larger outbreaks, affecting approximately 100 people, were most likely caused by preformed bacterial toxins in foods (Clostridium perfringens and Bacillus cereus).

In late June, a diarrhoeal outbreak affecting approximately 70 residents of a nursing home in the Blue Mountains was reported. The epidemiological and clinical picture of the outbreak indicated it was most likely foodborne, with C. perfringens toxin detected in several stool samples. The organism was not detected in food samples. 
Figure 1. Reports of selected communicable diseases, NSW, January 2004 to June 2008, by month of onset.

Preliminary data: case counts in recent months may increase because of reporting delays.

Laboratory-confirmed cases only, except for measles, meningococcal disease and pertussis.

BFV, Barmah Forest virus infections; RRV, Ross River virus infections; lab conf, laboratory confirmed;

Men $\mathrm{Gp} C$ and $\mathrm{Gp} \mathrm{B}$, meningococcal disease due to serogroup $C$ and serogroup $B$ infection;

other/unk, other or unknown serogroups.

NB: Multiple series in graphs are stacked, except gastroenteritis outbreaks.

NB: Outbreaks are more likely to be reported by nursing homes and hospitals than by other institutions.

\begin{tabular}{|lr|}
\hline \multicolumn{2}{|c|}{ NSW Population } \\
Male & $50 \%$ \\
$<5$ y & $7 \%$ \\
$5-24$ y & $27 \%$ \\
$25-64$ y & $53 \%$ \\
$65+y$ & $13 \%$ \\
Rural & $46 \%$ \\
\hline
\end{tabular}
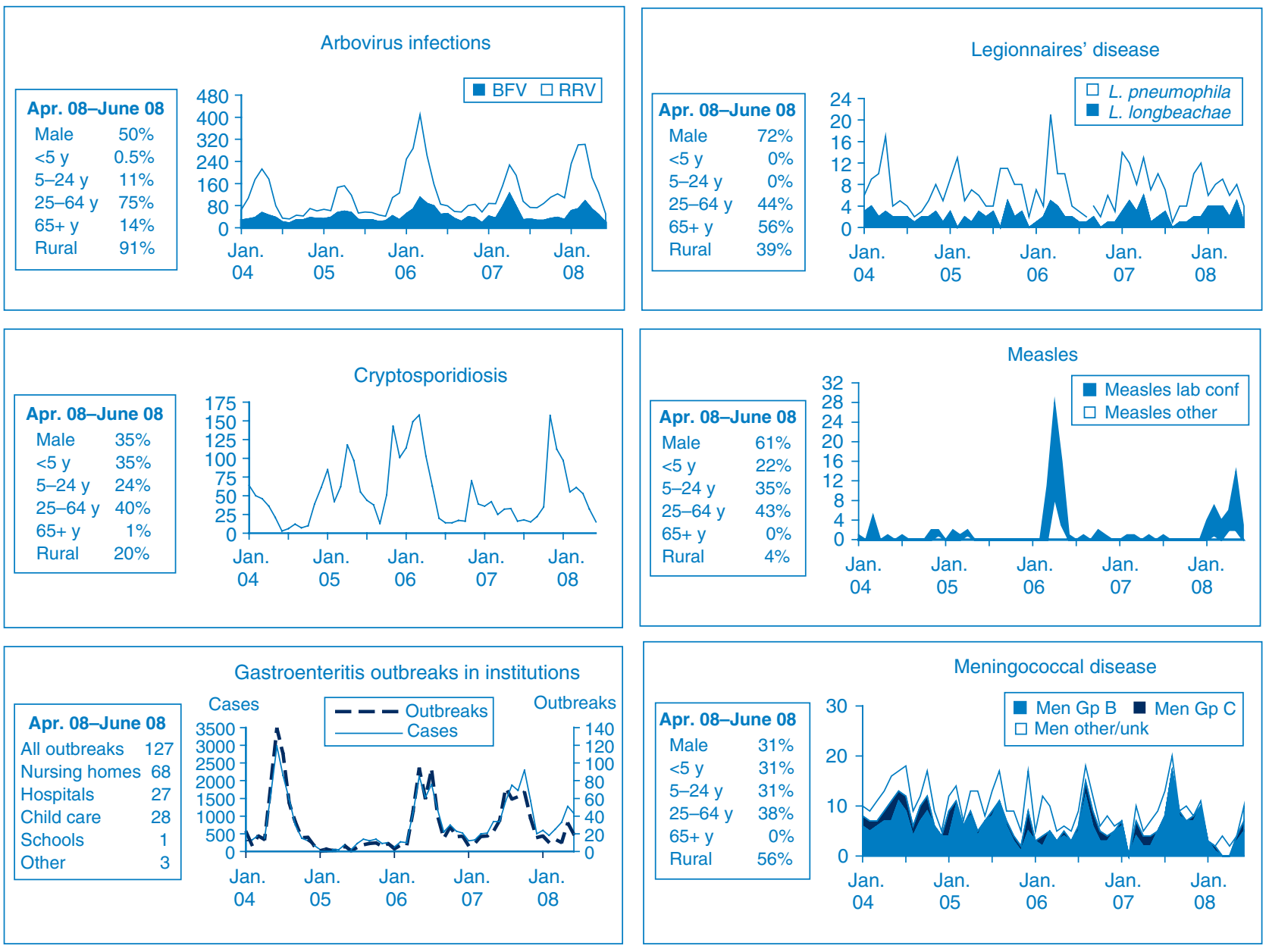
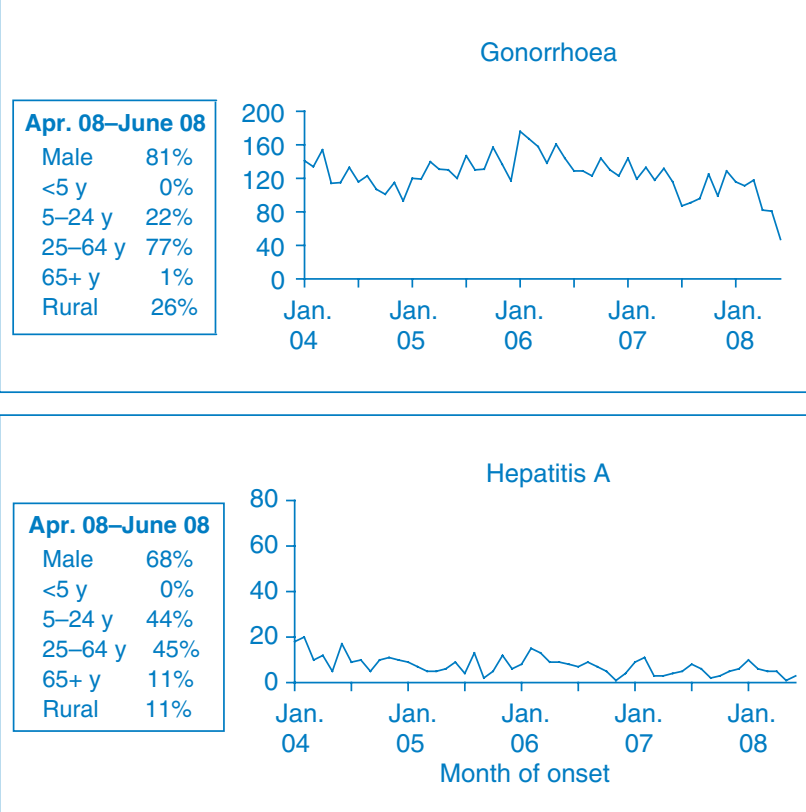
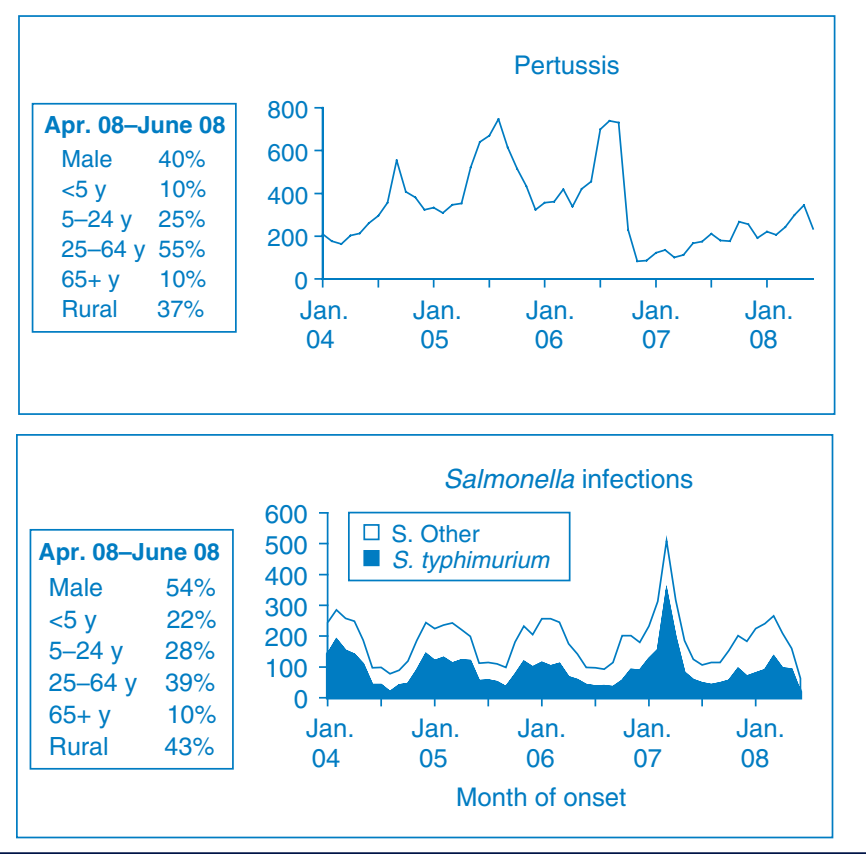


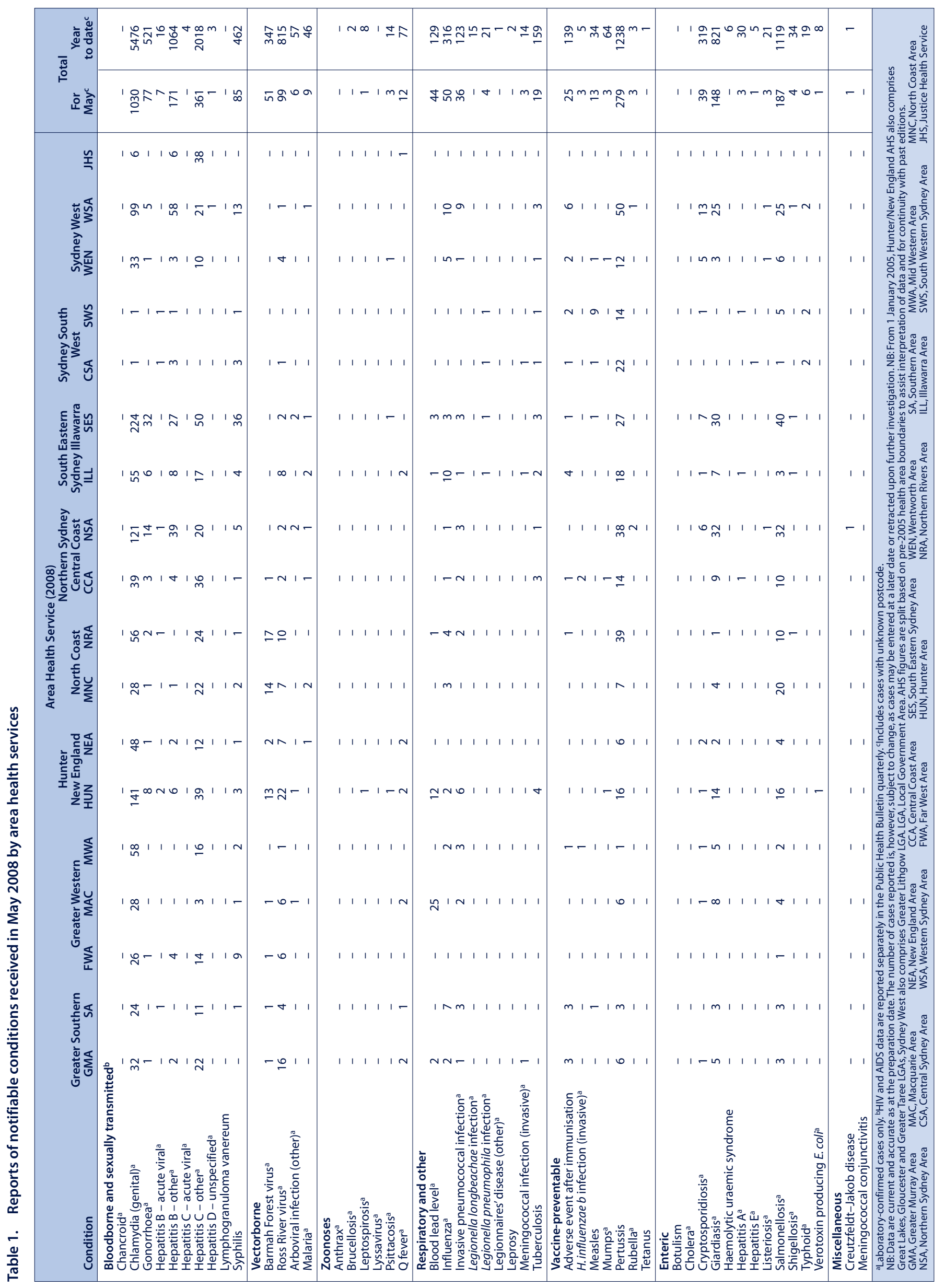




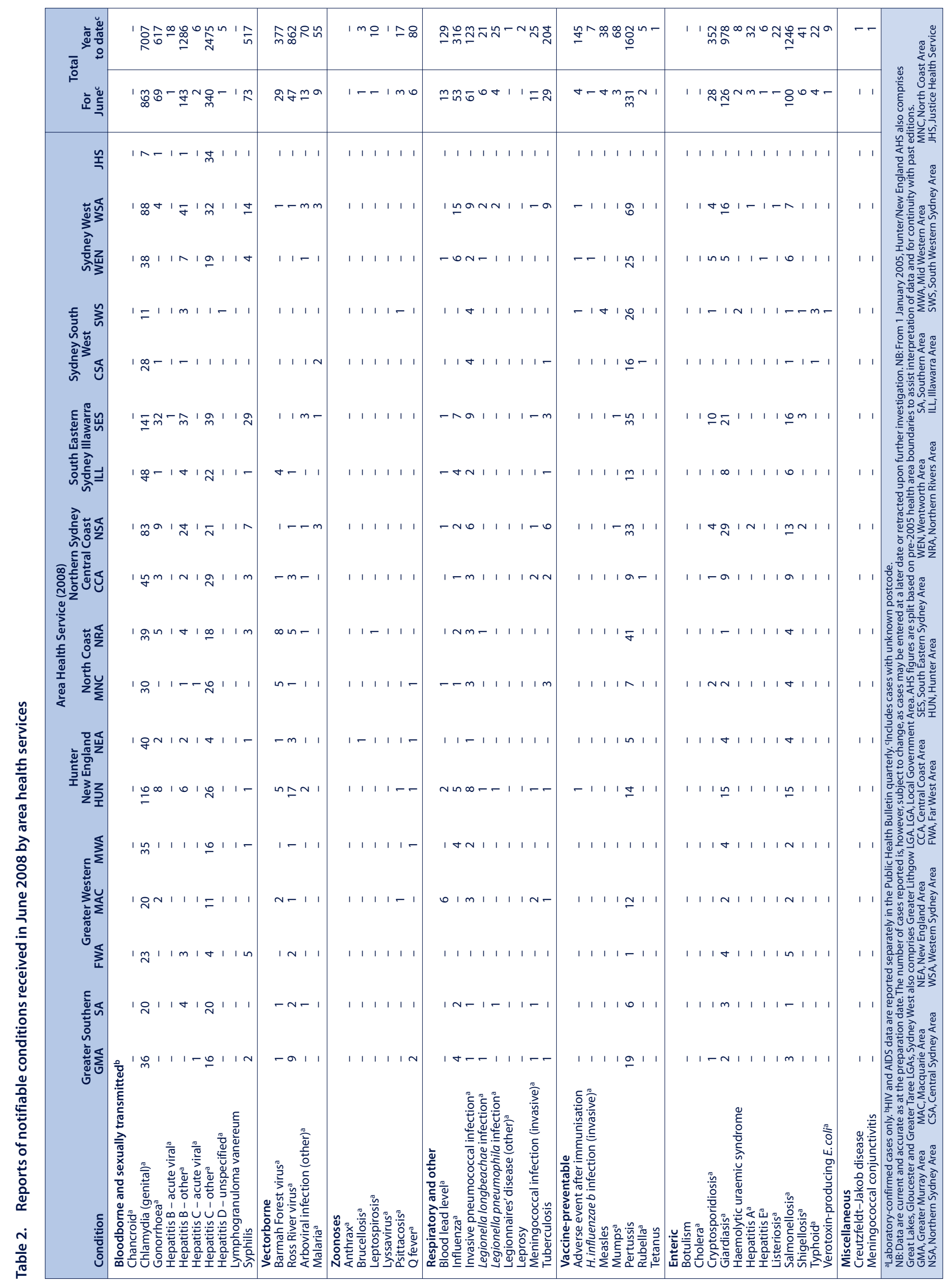

